\title{
A DICHROMATIC POLYNOMIAL FOR WEIGHTED GRAPHS AND LINK POLYNOMIALS
}

\author{
LORENZO TRALDI
}

(Communicated by Thomas Brylawski)

\begin{abstract}
A dichromatic polynomial for weighted graphs is presented. The Kauffman bracket of a signed graph, an invariant inspired by the Jones polynomial of a link in three-space, is shown to be essentially an evaluation of this dichromatic polynomial, as are the homfly polynomials of certain particular types of links.
\end{abstract}

\section{THE DiChROMATIC POLYNOMIAL OF A WEIGHTED GRAPH}

In this note we use the term graph to refer to a finite multigraph, i.e. a graph $G=(V(G), E(G))$ which has a finite number $n>0$ of vertices and a finite number $m \geq 0$ of edges, some of which may be multiple edges or loops. The zeroth Betti number $p_{0}(G)$ of such a graph is the number of its connected components, and the first Betti number $p_{1}(G)$ is $p_{1}(G)=m-n+p_{0}(G)$. A weighted graph is a graph $G$ together with a function $w$ mapping $E(G)$ into some commutative ring with unity $R$; if $e$ is an edge of $G$ then $w(e)$ is the weight or conductance of $e$.

If $S$ is any set of edges of a graph $G$ then we denote by $G: S$ the subgraph of $G$ which includes all the vertices of $G$ but only the edges in $S$. Using this notation, we define the dichromatic polynomial $Q(G ; t, z)$ of a weighted graph $G$ to be

$$
Q(G ; t, z)=\sum\left(\prod w(e)\right) t^{p_{0}(G: S)} z^{p_{1}(G: S)},
$$

the sum taken over the set of all subsets $S$ of $E(G)$ and the product taken over $S$. We should point out that despite calling this a "polynomial," we do not require that $t$ and $z$ be indeterminates; indeed, it is often convenient to treat them as elements of $R$. (See Proposition 1, for instance.)

It is obvious that the ordinary dichromatic polynomial of an unweighted graph [9, IX.1] can be recovered from this one by assigning all edges the weight 1 . It is also clear that this dichromatic polynomial is essentially a matroid invariant; we will not pursue this here, except to note that consequently $t^{-p_{0}(G)} Q(G ; t, z)$

Received by the editors April 15, 1988 and, in revised form, August 10, 1988.

1980 Mathematics Subject Classification (1985 Revision). Primary 05C99, 57M25.

Key words and phrases. Weighted graphs, dichromatic polynomials, classical links, bracket polynomials. 
is unchanged if $G$ is replaced by a weighted graph that is 2-isomorphic to $G$ in the appropriate sense (cf. [10, Chapter 6] for the unweighted case).

The proofs of the first five parts of the following theorem are entirely analogous to the proofs of similar results for the ordinary dichromatic polynomial of an unweighted graph [9, IX.1].

Theorem 1. $Q(G ; t, z)$ has the following properties.

(i) If $m=0, Q(G ; t, z)=t^{n}$.

(ii) If $G$ is disconnected, $Q(G ; t, z)$ is the product of the dichromatic polynomials of the components of $G$.

(iii) If $G$ has an edge $e$ that is not a loop, then $Q(G ; t, z)=Q(G-e ; t, z)+$ $w(e) Q(G / e ; t, z)$, where $G-e$ is the graph obtained from $G$ by deleting the edge $e$, and $G / e$ is the graph obtained from $G-e$ by identifying the vertices of $e$ (both carry edge weightings induced directly by those of $G)$.

(iv) If $e$ is a loop then $Q(G ; t, z)=(1+w(e) z) Q(G-e ; t, z)$.

(v) If $e$ is an isthmus then $Q(G ; t, z)=(w(e)+t) Q(G / e ; t, z)$.

(vi) If $w(e)=0$ then $Q(G ; t, z)=Q(G-e ; t, z)$.

A curious feature of this dichromatic polynomial is that except for the $n=1$ case of (i), both (i) and (ii) are unnecessary. For a disconnected graph can always be made connected, without affecting its polynomial, by inserting edges of weight zero; and the polynomial of this connected graph can be calculated without encountering a disconnected graph, simply by choosing to use (v) rather than (iii) whenever an isthmus is to be removed.

It is interesting to observe that this dichromatic polynomial does not have the same duality between the variables $t$ and $z$ as the unweighted version does, because of the different ways that the weights appear in (iv) and (v). Moreover, the variables $t$ and $z$ possess an interesting ambiguity: to a certain extent, they can be regarded either as essential features of the polynomial or as aspects of the edge weighting. To see this, note that if $z G$ is obtained from $G$ by multiplying all its edge weights by $z$, then $z^{n} Q(G ; t, z)=Q(z G ; t z, 1)$.

A useful feature of the dichromatic polynomial of a connected, unweighted graph is its expansion in terms of the activities of edges with respect to spanning trees [9, IX.6]. We recall the definition: given an arbitrary linear ordering of $E(G)$ and a spanning tree $T$ of $G$, an edge $e$ of $T$ is internally active with respect to $T$ if it precedes all other edges of $G$ whose end vertices lie in different components of $T-e$; and an edge $e$ not in $T$ is externally active with respect to $T$ if it precedes all the edges of $T$ that lie in the (unique) simple circuit determined by $T$ and $e$. The dichromatic polynomial of a weighted graph also has an expansion in terms of these activities, stated in

Theorem 2. Let $G$ be a connected, weighted graph. Then

$$
t^{-1} Q(G ; t, z)=\sum\left(\prod_{I I} w(e)\right)\left(\prod_{I A}(t+w(e))\right)\left(\prod_{E A}(1+z w(e))\right),
$$


the sum taken over the set of all spanning trees $T$ of $G$ and the three products taken (respectively) over the sets of edges that are internally inactive, internally active, and externally active with respect to $T$.

Note that Theorem 2 can be applied to any graph, if one inserts enough edges of weight zero to make the graph connected.

We close this section with two simple consequences of Theorem 2 .

Corollary 1. If $K(G)$ is the Kirchhoff matrix of $G$ [9, VI.4], $1 \leq r \leq n$, and $K_{r}(G)$ is obtained by deleting the $r$ th row and column of $K(G)$, then the determinant of $K_{r}(G)$ can be obtained from $t^{-1} Q(G ; t, z)$ by evaluating at $t=z=0$. Proof. Simply replace $t$ and $z$ by 0 in Theorem 2 (or in the definition of $Q$ ), and apply the matrix-tree theorem [9, VI.4].

Corollary 2. Let $r G$ be obtained from a weighted graph $G$ by multiplying all its edge weights by $r$. Then

$$
Q(r G ; t, z)=r^{n} Q\left(G ; t r^{-1}, z r\right) .
$$

(To avoid problems when $r$ is 0 or a zero divisor, the right-hand side of this equation should be interpreted as follows. If $x$ is an indeterminate then $x^{n} Q\left(G ; t x^{-1}, z x\right)$ is an element of the ring $R\left[t, z, x, x^{-1}\right]$ of polynomials in $t, z, x$, and $x^{-1}$ with coefficients from $R$. It happens that this element has no negative powers of $x$, so it's an element of $R[t, z, x] ; r^{n} Q\left(G ; t r^{-1}, z r\right)$ is to be the image of $x^{n} Q\left(G ; t x^{-1}, z x\right)$ under the map $R[t, z, x] \rightarrow R[t, z]$ that sends $x$ to $r$.)

\section{GeNERIC POLYNOMIALS}

In this section we present a distinctive feature of the dichromatic polynomial of weighted graphs, the existence of "generic" or "universal" polynomials.

The following is easily proven using Theorem 1 or the definition of $Q$.

Proposition 1. Let $e_{1}, \ldots, e_{k}$ be edges of $G$ that all have the same end vertices. Let $G^{\prime}$ be obtained from $G$ by replacing $e_{1}, \ldots, e_{k}$ by a single edge of weight

$$
z^{-1}\left(-1+\prod\left(1+w\left(e_{i}\right) z\right)\right)
$$

Then $Q(G ; t, z)=Q\left(G^{\prime} ; t, z\right)$.

Suppose $n>0$, and let $\left\{w_{i j}: 1 \leq i \leq j \leq n\right\}$ be an algebraically independent set of indeterminates. Let $G_{n}$ be the weighted graph with $V\left(G_{n}\right)=$ $\left\{v_{1}, \ldots, v_{n}\right\}$ such that whenever $1 \leq i \leq j \leq n, G_{n}$ has a single edge of weight $w_{i j}$ with end vertices $v_{i}$ and $v_{j}$. Then $Q\left(G_{n} ; t, z\right)$ is a polynomial (with integer coefficients) in $t, z$, and the various $w_{i j}$, and Proposition 1 implies

Theorem 3. If $G$ is any weighted graph with $n$ vertices, $Q(G ; t, z)$ can be obtained from $Q\left(G_{n} ; t, z\right)$ by appropriately evaluating the various $w_{i j}$. 


\section{KAUFFMAN'S BRACKET}

A signed graph is a graph $G$ together with a function $s$ mapping $E(G)$ into $\{+1,-1\}$. In [4] L. H. Kauffman introduced a "generalized Tutte polynomial for connected signed graphs," which we will call the Kauffman bracket and denote by $[G ; A, B, d]$; it is an integer polynomial in the indeterminates $A, B$, and $d$. The bracket is determined by the following two properties.

(i) If $G$ consists entirely of isthmuses and loops, then

$$
[G ; A, B, d]=(A+B d)^{i^{+}+k^{-}}(A d+B)^{i^{-}+k^{+}},
$$

where $i^{+}$is the number of isthmuses with sign $+1, k^{-}$is the number of loops with sign -1 , and so on.

(ii) If an edge $e$ of $G$ is neither an isthmus nor a loop, then $[G ; A, B, d]=$ $B[G-e ; A, B, d]+A[G / e ; A, B, d]$ if $e$ has sign +1 , while $[G ; A, B, d]=$ $A[G-e ; A, B, d]+B[G / e ; A, B, d]$ if $e$ has sign -1 .

Let $G$ be a connected signed graph. We associate to $G$ a weighted graph, also denoted $G$, whose underlying unweighted graph is the same as that of $G$. The edge weights to be used are elements of the Laurent polynomial ring $Z\left[A, A^{-1}, B, B^{-1}\right]$, and an edge of $G$ is assigned the weight $A B^{-1}$ or $A^{-1} B$ according to whether its sign is +1 or -1 .

Theorem 4. Let $G$ be a connected signed graph. Then the bracket of $G$ and the dichromatic polynomial of its associated weighted graph are related by

$$
d^{-1} Q(G ; d, d)=\left(\prod A^{(-1+s(e)) / 2} B^{(-1-s(e)) / 2}\right)[G ; A, B, d],
$$

the product taken over the set of all edges of $G$.

Proof. An inductive proof is easily given, using parts (iv) and (v) of Theorem 1 for part (i) of the definition of the bracket and part (iii) of Theorem 1 for part (ii) of the definition of the bracket.

Note that consequently, $[G ; A, B, d]$ is essentially a polynomial in only two variables, $d$ and $A B^{-1}$, with positive and negative powers of the latter.

Also, note that $Q(G ; t, z)$ is genuinely more sensitive than $[G ; A, B, d]$. For instance, if $G$ consists of two loops of opposite sign and $H$ consists of two isthmuses of opposite sign then $Q(G ; d, d)=Q(H ; d, d)$ and $[G ; A, B, d]=$ $[H ; A, B, d]$, but $Q(G ; t, z) \neq Q(H ; t, z)$.

Essential features of the dichromatic polynomial can be seen to correspond to particular properties of the bracket via Theorem 4. For instance, the expansion of the bracket as a sum indexed by states $[3,4]$ or spanning subgraphs [5] corresponds to the definition of the dichromatic polynomial in terms of the various subsets $S$ of $E(G)$. Also, the spanning tree expansion of the bracket $[4,8]$ corresponds to the expansion of the dichromatic polynomial given in Theorem 2, though the one given in Theorem 2 may be somewhat simpler in practice because it does not involve any contribution from externally inactive edges. 
Before closing this section, we should mention that K. Murasugi [6] has recently given an extensive analysis of a polynomial invariant of a signed graph $G$ that is essentially $t^{-1} Q(G ; t, z), G$ the weighted graph associated to the signed graph $G$ as above. He concentrates his attention on the terms of maximal and minimal degree in this polynomial, which are of great importance in the application of this material to link theory.

\section{LINKS IN THREE-SPACE}

A (tame) link in three-space is a collection of finitely many, pairwise disjoint, smooth simple closed curves; a link may be oriented by specifying orientations of these curves. A projection of such a link in the plane is regular provided that the only singularities of the projection are transverse double points ("crossings"), of which there are only finitely many; clearly such a projection is a regular fourvalent graph in the plane, possibly with additional "free loops" incident to no vertices. The link projection is split if this graph is disconnected. The link may be recovered from a regular projection provided that at each crossing, the choice of the overpassing arc is specified; this is usually done by deleting short arcs in the projection on each side of an underpass, resulting in a link diagram. Two links are of the same type provided that one can be manipulated into the other in space, without cutting; for oriented links the orientations must coincide after the manipulation. The fundamental problem of link theory is the problem of recognizing whether or not two given links are of the same type.

Given a link diagram $D$, the complementary regions (or faces) of $D$ in the plane can be colored in a checkerboard fashion, so that if the boundaries of two regions share an arc, then one of the regions is shaded and the other is not. (The unbounded face is usually left unshaded.) A signed graph $B(D)$ can be obtained from $D$ in the following way.

If $D$ is not split, $B(D)$ has a single vertex corresponding to each shaded face of $D$, and a single edge corresponding to each crossing, connecting the vertex (or vertices) corresponding to the shaded face(s) incident at that crossing. The edges are given signs according to the scheme illustrated in Figure 1.


FIGURE 1

If $D$ is split, then $D$ is the disjoint union of various nonsplit subdiagrams, and $B(D)$ is defined to be the disjoint union of the graphs associated to these subdiagrams. 
Kauffman's bracket was inspired by the Jones polynomial, a link type invariant of oriented links that is a multiple of the reduced bracket $\left[B(D) ; A, A^{-1}\right.$, $-A^{2}-A^{-2}$ ] by a monomial in $A$. The reduced bracket has been of dramatic use in settling several longstanding conjectures about links and their diagrams [3-6, 8]. Given Theorem 4, it is natural to hope that for some choice of edge weightings in $B(D)$, the dichromatic polynomial $Q(B(D) ; t, z)$ might be of some further use in link theory. Such usefulness is limited, though, by the following observation.

Suppose $D$ is not split, and note that the transformation of link diagrams illustrated in Figure 2 (a Reidemeister move of the second type) corresponds to the insertion of a pair of edges of opposite signs in $B(D)$, in one of the two ways illustrated.

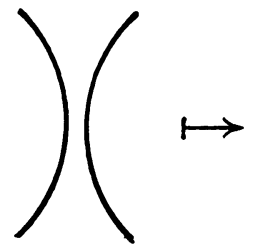

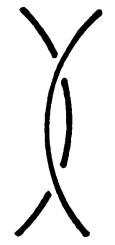

FIGURE 2

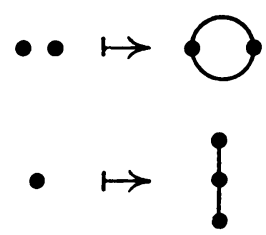

$\vdots$

Suppose that the edges of $B(D)$ are assigned weights in such a way that these two edges are weighted $x$ and $y$. If an evaluation of $Q(B(D) ; t, z)$ is to remain unchanged under the insertion of these two edges, a simple calculation suggests that under the evaluation we must have $t=z=-x-y$ and $x y=1$, and so this evaluation of $Q(B(D) ; t, z)$ must coincide, up to a normalization factor and a change of variables, with the reduced bracket or some evaluation of it.

This does not absolutely rule out the possibility that some evaluation of $Q(B(D) ; t, z)$ other than the reduced bracket may be of use in link theory, though, for such an evaluation might not be absolutely invariant under the Reidemeister moves, but might vary in a sufficiently simple way that a significant amount of information about link type could be recovered from it.

A less conventional relationship between link diagrams and graphs has been suggested by F. Jaeger [2]; we close with a generalization of this relationship and the principal theorem of [2]. We should mention that the original version of this paper contained a significantly milder generalization of Jaeger's theorem, involving only the upper two portions of Figure 3. The one included below is essentially due to T. Przytycka and J. Przytycki [7]; we are grateful to them for drawning it to our attention.

Suppose we are given a plane graph $G$, i.e. a graph that consists of particular points and arcs in the plane, rather than abstract vertices and edges. Suppose further that each edge of $G$ carries one of four possible weights in the Laurent polynomial ring $Z\left[x, x^{-1}, y, y^{-1}, z, z^{-1}\right]: y / z, x / z, z / y$, or $z / x$; and let $a, b, c$, and $d$ be the numbers of edges of each of these weights (respectively). Construct a modified medial graph $M^{\prime}(G)$ as follows. If $G$ consists solely of 
a single vertex then $M^{\prime}(G)=G$. If $G$ is connected and has some edges then $M^{\prime}(G)$ has a vertex on each edge of $G$, and its edge set consists of pairwise disjoint circuits, one running around the boundary of each face of $G$ (the edges of such a circuit should lie within the corresponding face of $G$ ). If $G$ is not connected, then $M^{\prime}(G)$ is the disjoint union of the modified medial graphs of its components. We can obtain a diagram $D(G)$ of an oriented link from $M^{\prime}(G)$ by replacing each isolated vertex of $M^{\prime}(G)$ with a small circle (of arbitrary orientation), and replacing each nonisolated vertex of $M^{\prime}(G)$ with a clasp as in Figure 3; in Figure 3 the shaded regions are those that contain the edge of $G$ on which the indicated vertex of $M^{\prime}(G)$ lies. The portions of $D(G)$ arising from different components of $M^{\prime}(G)$ should be disjoint.

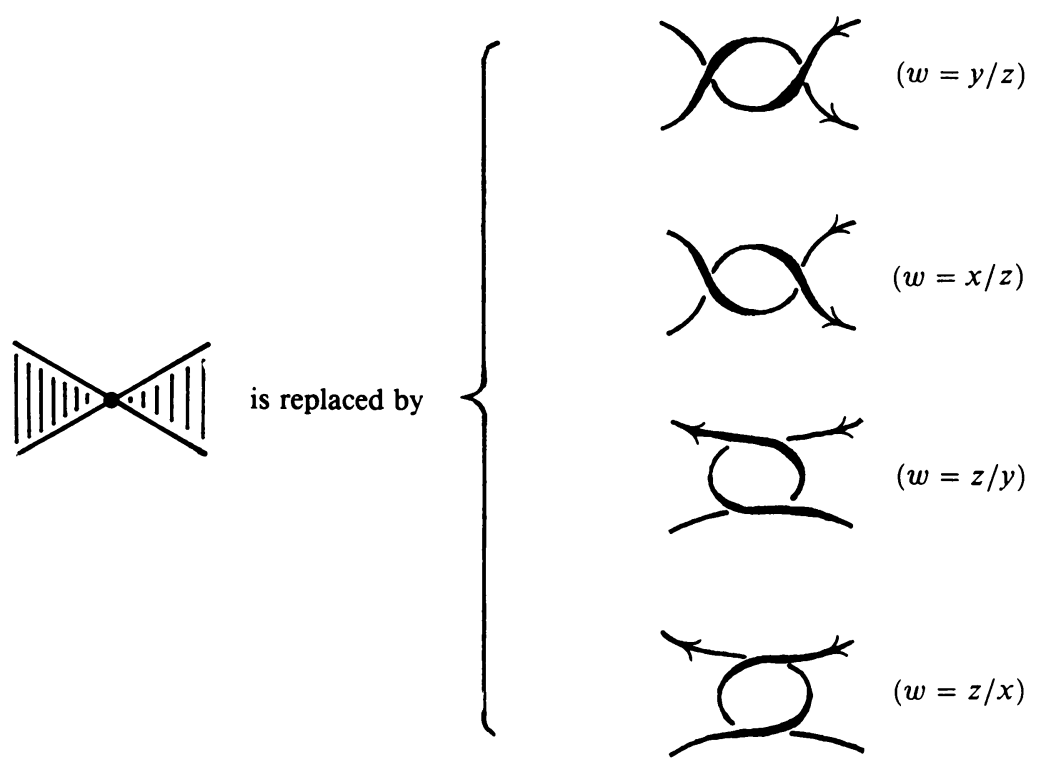

FiguRe 3

In [2] Jaeger proved that in case $G$ is connected and all its edges have weight $y / z$, the homfly polynomial $P(D(G) ; x, y, z)$ [1] is a multiple of an evaluation of the Tutte polynomial of the unweighted graph $G$. (The Tutte polynomial, or dichromate, is a simple variant of the dichromatic polynomial $[9$, IX.6], and the homfly polynomial is a link type invariant of oriented links that specializes to the Jones polynomial and is determined by these two properties: $P(L ; x, y, z)=1$ if $L$ is a single unknotted circle; and $x P\left(L_{+} ; x, y, z\right)+y P\left(L_{-} ; x, y, z\right)+$ $z P\left(L_{0} ; x, y, z\right)=0$ if $L_{+}, L_{-}$, and $L_{0}$ possess diagrams that differ only in a single location as in Figure 4.)

The argument of [2] can be generalized directly to prove 


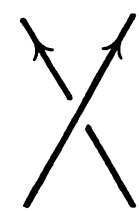

$L_{+}$

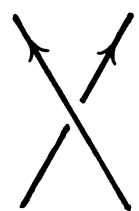

$L_{-}$

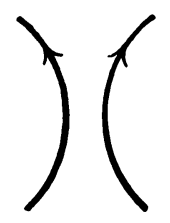

$L_{0}$

FIGURE 4

Theorem 5. Let $G$ be a plane graph with four possible edge weights, $D(G)$ the associated link diagram described above. Then the homfly polynomial $P(D(G)$; $x, y, z)$ and the dichromatic polynomial of the weighted graph $G$ are related by

$$
\begin{gathered}
(-x / z)^{a}(-y / z)^{b}(-x / y)^{c}(-y / x)^{d} P(D(G) ; x, y, z) \\
=(-(x+y) / z)^{-1} Q(G ;-(x+y) / z,-(x+y) / z) . \\
\text { ACKNOWLEDGMENTS }
\end{gathered}
$$

\section{ACKNOWLEDGMENTS}

We are grateful to G. Gordon for many enlightening conversations about polynomial invariants of graphs and matroids, and to T. Przytycka and J. Przytycki for mentioning their version of Theorem 5 to us. We also thank the referee for many useful suggestions.

Added in proof. Several months after the present paper was accepted for publication in these Proceedings, we read On the random-cluster model by C. M. Fortuin and P. W. Kasteleyn [Physica 57 (1972), 536-564]. In that paper a polynomial invariant of doubly weighted graphs (graphs in which there are two edge weights, say $w$ and $w^{\prime}$ ) was introduced, in the context of probabilistic graphs used to represent several types of mechanical systems. The dichromatic polynomial we have discussed here is essentially the special case $w^{\prime} \equiv 1$ of this doubly weighted polynomial, and the generalization of Theorem 1 to this doubly weighted polynomial appeared in the earlier paper. We hope the reader will give Professors Fortuin and Kasteleyn due credit for their contributions.

\section{REFERENCES}

1. P. Freyd, D. Yetter, J. Hoste, W. B. R. Lickorish, K. Millett, and A. Ocneanu, A new polynomial invariant of knots and links, Bull. Amer. Math. Soc. 12 (1985), 239-246.

2. F. Jaeger, On Tutte polynomials and link polynomials, Proc. Amer. Math. Soc. 103 (1988), 647-654.

3. L. H. Kauffman, State models and the Jones polynomial, Topology 26 (1987), 395-407.

4. __ New invariants in the theory of knots, Amer. Math. Monthly 95 (1988), 195-242.

5. K. Murasugi, Jones polynomials and classical conjectures in knot theory, Topology 26 (1987), 187-194.

6. $\ldots$, On invariants of graphs with applications to knot theory, Trans. Amer. Math. Soc. (to appear).

7. T. Przytycka and J. Przytycki, Signed dichromatic graphs of oriented link diagrams and matched diagrams, notes, Univ. of Toronto; 1987.

8. M. B. Thistlethwaite, A spanning tree expansion for the Jones polynomial, Topology 26 (1987), 297-309.

9. W. T. Tutte, Graph theory, Cambridge Univ. Press, Cambridge, 1984.

10. N. White (ed.), Theory of matroids, Cambridge Univ. Press, Cambridge, 1986.

Department of Mathematics, Lafayette College, Easton, Pennsylvania 18042 